\title{
Reciprocal Adaptation and Emotion in Conflict Transformation
}

\author{
Bilyana Martinovski \\ School of Business and Informatics, Boras University College, Sweden
}

\begin{abstract}
The present paper explores reciprocal adaptation of emotion in conflict transformation and negotiation (Martinovski and Mao 2009, Martinovski 2010) by studying authentic discourse. It finds that reciprocal adaptation affects emotion in conflict transformation. It is most evident in empathy exchanges and realizes as direct and opposite reciprocity. The study concludes that conflict is a method through which participants learn how to enter into each other's frame of reference.
\end{abstract}

\section{Introduction}

Management of conflict, including conflict transformation, involves management of emotion in the process of problem reframing and evolution of problem representations, including goals, values, criteria, and preferences (Shakun 1991, Kumar 1997, Barry 2008, Druckman \& Olekalns 2008, Martinovski 2010). At the same time, there are discoursive mechanisms such as reciprocal adaptation (Gumperz 1982), which influence participant's frames of reference. In the context of conflict management, reciprocal adapatation may work both ways, it may lead to a stalemate or to mutual cooperation. The purpose of this paper is to explore how reciprocal adaptation influences emotion in conflict management and what linguistic phenomena and argumenation are involved in the process. The study uses discourse analysis of linguistic manifestations of reciprocal adaptation and emotion in face-to-face two-party friendly exchanges and a three-party plea bargain negotiation from Heritage's and Maynard's corpora.

\section{Reciprocal adaptation in empathy exchange}

Gumpertz (1982: 13) defines reciprocal adaptation as "the procedure ... where each participant gradually learns to adapt and to enter into the other's frame of reference." This definition does not involve emotion, however we find reciprocal adaptation most evident in social empathy interplay. The following example illustrates a successful ritualistic 'empathic moment' (Heritage, 2005). The transcription conventions are: '[ ]' stands for overlapped speech; ':' stands for prolonged vowel; '=' stands for latching speech; '/' indicates pause; capital letters indicate emphatic speech; '+' indicates cut-off; '( )' stands for inaudible speech; '?' stands for rising intonation; '.' stands for falling intonation; ',' stands for continuing intonation; Each 
line in the transcription indicates an intonation unit; $\{0.9\}$ stands for seconds of pause. The empathy episode starts with an announcement of trouble on line 2, welcomed and elicited on line 3. Turn 14 gives the punch line, which elicits empathy.

Example 1:

1. Joy: ye-:s I'm alright,

2. Les: oh:. hh yi-m- you know I-I- I'm boiling about something hhhheh [1 heh hhhh]

3. Joy: [1 wha::t.]

..

14. Les: AND uh we were looking rou-nd the sta:lls 'n poking about 'n he came up t'me 'n be said Oh: hhello leslie, . still trying to buy something f'nothing,

15. Joy: .hhhahhhhhh! $\{0.8\}$ oo[5: : :: LESLI E]

16. Les: [5 oo:.ehh heh heh ] $\{0.2\}$

17. Joy: i:s [6 n't he]

18. Les: [6 what] do you sa:y. $\{0.3\}$

19. Joy: oh isn't he drea:dful.

20. Les: eye-:-:s: $\{0.6\}$

21. Joy: what'n aw::f'l ma::[7:::n]

22. Les: [7 ehh] heh-heh-heh

23. Joy: oh:: honestly, I cannot stand the man it's $\backslash$ just $\{$ no[ $[8 ;]$

...

26. Les: $=[9$ heh-heh he-e $]$ uh: eh [10 eh hhhhh]

27. Joy: $=[10 \mathrm{O} H::::$.$] I do think he's dreadful$

28. Les: tch oh: dea-r

29. Joy: oh: he r[11 eally i ] :s,

Reciprocal adaptation is manifested linguistically when Joy gives her rather emotional empathy on line 15 and Les implicitly accepts it on line 16 . The exclamation forms on lines 15 -16 as well as $26-27$ are similar, in that sense the speakers mirror or imitate each other, although the functions of the utterances are different.

\section{Reciprocal adaptation as conflict management}

In conflict situations we observe escalation of conflict or gradual resolution as a result of reciprocal adaptation, which in the analysed plea bargain involves not only factual references 
but also emotional learning. Emotion is thus an active force in transformation of negotiation from cooperation to conflict and from conflict to cooperation and reciprocal adaptation is the procedure through with it operates in discourse. In the observed data, the emotional transformation chain consists of stages of joint contributions (Clark 1999) where each participant gives a new emotional direction or potential of negotiation, towards or away from conflict:

1. flattery is reciprocated with confidence resulting in openness for cooperation

2. laughter, carrying sense of superiority is reciprocated with seriousness, confusion, reluctance

3. ridicule, superiority and bonding is reciprocated with confusion, angst, defensiveness, uncertainty, self-criticism resulting in limited concessions

4. elicitation of empathy is reciprocated with refusal of empathy and irony resulting in challenge

5. aggression and threats are reciprocated with confusion, anxiety, rebuts resulting in conflict

6. breakdown is expressed by reciprocal silence

7. exchange of empathy regarding other issue results in reconciliation and friendly agreement

The plea bargain starts with cooperation, moves through confusion, reluctance, helplessness, and challenging to conflict, and then through diversion and reconciliation ends with cooperation. We observe direct reciprocal adaptation in choices of lexical style, intonations, and turn-taking in the initial and final coopeative stages of the plea bargain but we also notice opposite reciprocal adaptation manifested by opposite emotional expressions in the middle stage. Opposite reciprocity evolves into aggression and a stalemate. Defence counsel (Def) interprets Prosecutor's (Prs) stance taking as a challenge (lines 204-206) and responds with sudden explosive counter challenge. The entertaining and ridiculing style, interchanged with demonstrations of helpfulness and agreeability develops into an expression of anger, disgust, contempt and a decisive threat (line 207). Prs' reacts with self-explanation presented in a weak manner as he stutters and has difficulties formulating a sentence (line 209). Def continues his ridicule by mocking back channels, initial interruptions, latching, ridiculing mocking repetitions, etc. (lines, 207, 208, 216, 220). In this way, Def gains a dominant emotional role in the negotiation, wins the floor and presents his personal hypothetical interpretations as arguments.

Example 2.

204 Prs: He has ub a: one prior. (0.3) conviction in this jurisdiction

205 with thee uhm (0.8) sheriff's office, of of interestinly 
206 enough. u:v striking a public officer and of disturbing peace.

207 Def: Will you knock it off. ((disgusted tone)) (0.5) You wanna make

208 a federal case out of this;

209 Prs: N:o, [I I just] think [that that i]t's it's not uh this uh=

210 Def: [ $\quad \mathrm{hhh}] \quad[\mathrm{h} \mathrm{h} \mathrm{m} \mathrm{]}$

211 Prs: =happy go lucky chap's uh first (1.0) encounter with uh um (1.8)

212 Def: [Statistic]ly if ya got black skin:. you ar $(0.2)$ you ar $()=$.

213 Prs: $[(\quad)]$

214 Def: $=$ hhighly likely to contact the police. I think

215 uh:substantially more likely than if you're white. $<$ Now come

216 on. $<$ Whadda want from 'im. (0.6) He's got a prior.

$217 \quad(1.8)$

218 Jge: Well we know he spent ten ho:urs, ehhem (1.0) end

219 uh:: [we know he's been down here fer] mo:re

220 Def: $\quad[(\mathrm{He}) \underline{\mathrm{o}}: \mathrm{n} 1$ y s p e $\mathrm{n} \underline{\mathrm{t}}$ ten] $(($ mock shock $))$

$221 \quad(0.8)$

222()$: \quad(($ throat clear $))=$

Emotionally loaded imperative expressions such as 'knock it off', 'come on' and throat clearing act as more powerful persuasion devices than the arguments, which are inferential and unmotivated. Def's sudden anger display has a successful strategic effect (Vogel 2008). Prs' emotive-cognitive reaction/adaptation to threats and anger is expressed by increase of self-repetitions, pauses, hesitation sounds, and ends with silencing. The emotional transformation here is: aggression, threats $->$ confusion, anxiety, rebuts $=$ conflict. This form of adaptation is reciprocal but opposite. Example 3 below illustrates direct reciprocal adapation which constitutes the final agreement and resolution of conflict (lines 239-243). It reminds of the adaptation in Example 1 as it is another form of empathic exchange (226-234).

Example 3.

225 Jge: Do I hear it raining again? Is it $\left[\left(\begin{array}{l}\quad)\end{array}\right]\right.$

226 Def:: $\quad\left[{ }^{\circ} \mathrm{Oh}\right.$ my] god.

$227 \quad(1.2)$

228 (D): $\quad \cdot h[h$

229 Jge: [I think that's rain [isn' it?

230 Def: $\quad$ [It only does it for spite. 
232 Prs: I think it is too. $=$

233 Def: $\quad=$ The suit's made of sugar. $<$ It melt[s.

234 Prs: $\quad[(\quad)$ out of $()$.

235 of (0.7) top on it. ${ }^{\circ}$ It's a firebird. It's a- (0.5) ((clicking

236 sound: chair?)) ( ).

237 ( ): ((audible breathing))

$238(\mathrm{~J}): \quad$ hhh

239 Prs: Is a seventy ${ }^{\wedge}$ five dollar (fine)?

240 Jge: Hh Heh huh. 'hh-

241 Def: Why don't we compromise and make it fifty.

242 Prs: That's done.

243 Def: $\quad \operatorname{Ar}[$ ri(h)ght.]

In this exchange, the interaction is harmonious, agreements and complaints are done in synchrony and quickly as latching utterances, Prs is not self-repeating, no hesitation sounds, no pauses. After this moment of reciprocal adaptation through empathy and common ground in relation to the rain, Prs makes his settlement offer in line with Def's desire. After an explosive negative emotional development, the reciprocal re-framing of the situation helped the participants' to restructure the value of the issue at stake and radically change preferences.

\section{Conclusions}

Emotional energy is not lost in communication, only transformed and reciprocal adaptation is a driving force in the process of problem restructuring, including goals, values, and preferences in the discussed negotiation. Reciprocal adaptation manipulates not only cognitive representations and views but also emotions. Negotiation evolves through joint emotional interactive expressions, evocations and interpretations. Linguistic features indicate reciprocal adaptation and emotional potential of utterances/phases, including their transformative power. Joint negative emotion projects and opposite reciprocal adaptation bring conflict whereas empathy, direct reciprocal adaptation and distance taking bring reconciliation and cooperation. Since reciprocal adaptation is a process of discoursive learning, both types of adaptation are forms of learning. In that sense, conflict is a method through which participants gradually learn how to enter into each other's frames of reference, which involves evolution of emotion and problem representation. 


\section{REFERENCES}

Barry, B. 2008. Negotiator affect: the state of the art (and the science). Group Decis Negot. J. 17, 97-105.

Clark, H.H. 1999. Using Language. Cambridge: Cambridge University Press.

Druckman, D. \& Olekalns, M. 2008. Emotions in negotiation. Group Decision and Neg. J.17, $1-11$.

Gumperz, Johan. J. 1982. Discourse Strategies. Cambridge University Press.

Heritage, J. (2005) Empathic Moments. Talk at CLIC/UCLA: California.

Kumar, R. 1997. The role of affect in negotiations: an integrative overview. J. of Appl Beh Sci. 33, 84-100.

Martinovski, B. \& Mao, W. 2009. Emotion as an argumentation engine: Modeling the role of emotion in negotiation. Group Decis Neg. J. 18, 235-259.

Martinovski, B. 2010. Emotion in negotiation. In Kilgour M. \& Eden C. (Eds.) Handbook of Group Decision and Negotiation. Springer.

Vogel, M. 2008. Coercion to Compromise. Oxford: Oxford University Press.

Shakun, M.F. 1991. Airline Buyout: Evolutionary Systems Design and Problem Restructuring in Group Decision and Negotiation. Man. Sci. 37, 1291-1303. 